\title{
Análise da assimetria nos padrões fundamentais arremessar e chutar em crianças
}

Ilca Santos ${ }^{1}$

Guilherme Lage ${ }^{1,2}$

Adriana Calvacante ${ }^{3}$

Herbert Ugrinowitsch ${ }^{3}$

Rodolfo Benda ${ }^{3}$

https://doi.org/10.5628/rpcd.06.02.188

\section{RESUMO}

A assimetria de desempenho nos padrões fundamentais arremessar e chutar foi analisada em 46 crianças, de ambos os sexos, entre 6 e 7 anos de idade. As crianças executaram 3 tentativas do arremesso e do chute com cada membro. O desempenho dos membros contralaterais foi analisado qualitativamente através do protocolo de McClenaghan e Gallahue (7). As análises do número de observações dos padrões fundamentais arremesso e chute indicaram efeitos de assimetria nos estágios inicial, elementar e maduro. Os resultados foram discutidos em termos de uma necessidade de desenvolvimento bilateral dos padrões fundamentais na infância.

Palavras-chave: padrões motores fundamentais, assimetria, arremesso, chute.
${ }_{1}^{1}$ Faculdades Unidas do Norte de Minas

2 Universidade FUMEC

${ }^{3}$ Universidade Federal de Minas Gerais

Brasil

\begin{abstract}
Asymmetry analysis of overarm throwing and kicking motor patterns in children

Performance asymmetry in the fundamental motor patterns of the overarm throwing and kicking was analyzed in 46 children aged 6 and 7 years old. Children performed 3 trials of both the overarm throwing and kicking with each limb. Performance of contralateral limbs was analyzed on the basis of qualitative analysis, as proposed by McClenaghan \& Gallahue (7). The inferential analyses of the number of observations in each component involved in the fundamental patterns of the overarm throwing and kicking indicated effects of asymmetry in the initial, elementary and mature stages. The findings are discussed in terms of necessity of a bilateral development of the fundamental motor patterns in childhood.
\end{abstract}

Key Words: fundamental motor patterns, asymmetry, overarm throwing, kicking. 


\section{INTRODUÇÃO}

As assimetrias encontradas no comportamento motor apresentam duas dimensões. Uma das dimensões, conhecida como assimetria lateral de preferência, diz respeito à maior freqüência de utilização de um determinado membro comparado ao membro contralateral. Esta preferência se relaciona ao conforto e a segurança que um sujeito apresenta na execução de habilidades motoras específicas. A outra dimensão, definida como assimetria lateral de desempenho, refere-se à diferença na qualidade da execução das tarefas pelos membros contralaterais. O desenvolvimento dessas assimetrias encontra-se na interação entre fatores biológicos e ambientais. Em relação ao aspecto biológico a especialização hemisférica apresenta uma função predominante nas assimetrias. Entende-se que o hemisfério esquerdo é o responsável pelo processamento das informações relacionadas aos aspectos seqüenciais e temporais do movimento, enquanto o hemisfério direito lida mais eficientemente com o processamento paralelo e a percepção de aspectos espaciais do ambiente $(2,3)$. Já em relação à participação do ambiente na emergência dessas assimetrias, os aspectos culturais têm sido apontados como um dos principais fatores. De forma geral, o mundo ocidental tem sido construído por uma tendência à direção direita-esquerda. Os seres humanos nutrem estas assimetrias quando uma variedade de objetos é construída de acordo com essa tendência (12). Dessa forma, o tipo de prática vivenciada é um dos marcos para o desenvolvimento dessas assimetrias (8).

No ciclo de vida do ser humano, podem ser observadas já no início de seu desenvolvimento as assimetrias laterais de desempenho e preferência $(4,9)$. De acordo com Haywood (6), a preferência por um determinado membro já pode ser observada a partir dos 4 anos e se mantém estável durante a infância. Teixeira e Gasparetto (10) investigaram as assimetrias laterais no padrão fundamental arremesso em grupos de crianças entre 4 e 10 anos e observaram que, independentemente do aumento da proficiência na execução do arremesso com o avanço da idade, os níveis de assimetria de desempenho entre membros se mantêm na infância.
Comparativamente aos estudos sobre assimetria que analisaram o desempenho entre membros superiores, apenas um pequeno número de estudos tem investigado os efeitos da assimetria entre membros inferiores (1). Em relação ao padrão fundamental chutar em crianças, não foi observado na literatura estudos que investiguem esses movimentos de manipulação. Teixeira et al. (11) encontraram, de forma geral, um desempenho superior do membro dominante em relação ao não-dominante em adolescentes, em tarefas motoras relacionadas ao futebol. Os índices de assimetria encontrados foram diferentes entre as tarefas, assim como não apresentaram alta correlação entre eles. Isto evidencia o caráter dinâmico do desenvolvimento das assimetrias, que depende de aspectos como a idade, a quantidade de prática e as características específicas das tarefas. O desenvolvimento motor caracteriza-se como uma progressão de movimentos mais simples para habilidades motoras mais complexas e organizadas. Durante a fase motora fundamental, que compreende as idades entre 2 e 7 anos, há uma progressão que pode ser subdividida em três estágios: inicial, elementar e maduro (5). A aquisição destes padrões fundamentais é de essencial importância para o domínio das habilidades motoras. Se os padrões fundamentais formam a base do repertório motor através do qual o ser humano interage com o seu meio, justificam-se novos estudos sobre as assimetrias laterais nos padrões fundamentais. Tendo em vista que poucas investigações foram realizadas, novos conhecimentos acerca desse fenômeno podem, futuramente, auxiliar na compreensão sobre como as assimetrias afetam o desenvolvimento global das crianças no que tange às suas percepções sobre o mundo e sobre si mesmas. Dessa forma, o objetivo do presente estudo foi analisar os efeitos da assimetria lateral de desempenho nos padrões fundamentais arremessar e chutar em crianças.

\section{MÉTODO}

\section{Amostra}

A amostra foi composta por 46 crianças pré-escolares, destras, com idade de 7 anos (média de 84,6 meses de idade), de ambos os sexos. A participação das crianças teve o consentimento livre e esclarecido dos pais. 


\section{Instrumentos e tarefa}

Foram utilizados uma câmera filmadora digital (2.5 zoom, 3.2 megapixel, marca olympus), 1 tripé para apoio da câmera, 3 bolas de tênis, 3 bolas de futsal infantil. Todas as crianças executaram 3 arremessos com bolas de tênis e três chutes com bolas de futsal infantil com cada membro. A meta das tarefas foi arremessar e chutar "o mais longe possível". Para a análise dos padrões arremesso e chute foi utilizado o protocolo de avaliação de padrões fundamentais de McClenaghan e Gallahue (7).

\section{Procedimentos}

A câmera de vídeo foi posicionada a $45^{\circ}$ em relação a um ponto demarcado de execução. O critério adotado para a definição dos membros dominante e nãodominante, para cada criança, foi baseado na autodefinição das crianças sobre o membro com que elas gostavam de arremessar e chutar, e na definição de duas profissionais de Educação Física que trabalhavam com as mesmas. As crianças foram filmadas individualmente e sem a presença das demais no local reservado à coleta. Após as instruções padronizadas sobre como executar os movimentos fundamentais arremesso e chute e sobre a meta de arremessar e chutar o "mais longe possível", as crianças executaram três tentativas de cada movimento em ambos os lados. Inicialmente foram executados os arremessos e posteriormente os chutes. As tentativas foram contrabalançadas entre os membros esquerdo e direito. A análise dos movimentos foi realizada posteriormente por uma especialista em desenvolvimento motor.

\section{Tratamento dos dados}

A análise qualitativa dos movimentos foi realizada em termos de número de observações dos componentes propostos pelo modelo de McClenaghan e Gallahue (7) nos estágios inicial, elementar e maduro. Para a análise inferencial foi utilizado o teste de Mann-Whitney para comparar o número de observações entre os membros contralaterais em cada um dos estágios propostos. Foi adotado um nível de significância de 0,05 .

\section{RESULTADOS}

\section{Padrão fundamental arremesso}

O número de observações encontradas no componente braço pode ser observado na Figura 1. O teste de
Mann-Whitney indicou diferença significativa entre os membros direito e esquerdo no estágio elementar $[Z$ $(n=276)=-3,02, p<0,01]$ e no estágio maduro [Z $(\mathrm{n}=276)=3,75, p<0,01]$. Um maior número de observações foi encontrado para o membro esquerdo no estágio elementar, enquanto um maior número de observações foi encontrado para o membro direito no estágio maduro. Não foi encontrada diferença entre o número de observações entre os membros no estágio inicial $[Z(n=276)=-0,52, p=0,6]$.

\section{Arremessar - componente braço}

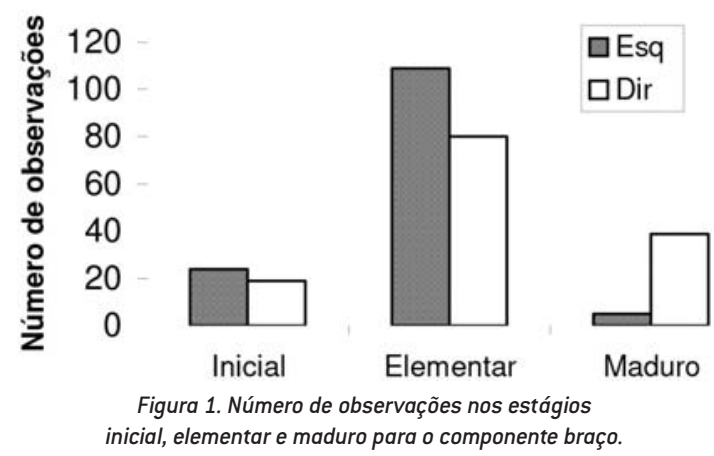

O número de observações encontradas no componente tronco pode ser observado na Figura 2. Na análise do componente tronco, foi encontrada diferença significativa entre o número de observações no estágio inicial $[\mathrm{Z}(\mathrm{n}=276)=-2,39, p<0,01]$, tendo um maior número de observações do membro esquerdo comparado ao membro direito. Não houve diferença significativa entre as observações para os estágios elementar $[\mathrm{Z}(\mathrm{n}=276)=1,04, \mathrm{p}=0,29] \mathrm{e}$ maduro $[Z(n=276)=1,36, p=0,17]$.

\section{Arremessar - componente tronco}

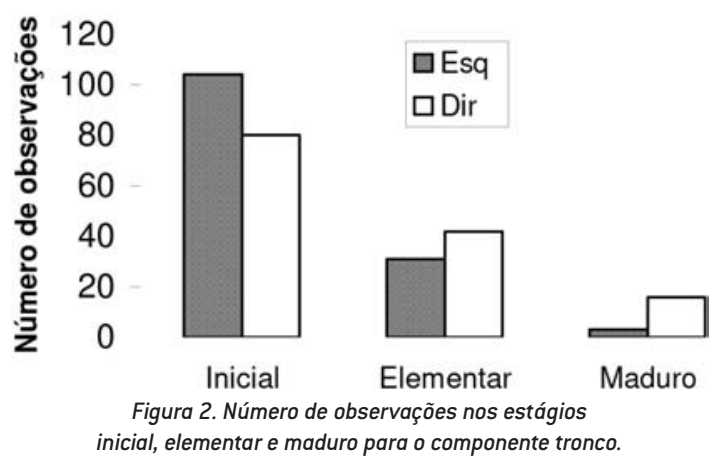


O número de observações encontradas no componente perna/pé pode ser visto na Figura 3. Na análise dos componentes foi encontrada diferença significativa para as observações nos estágios inicial [ $\mathrm{Z}$ (n $=276)=1,98, p<0,05]$, indicando um maior número de observações para o membro esquerdo quando comparado com o direito. Não foi encontrada diferença significativa para os estágios elementar $[\mathrm{Z}(\mathrm{n}=276)=-1,87, p=0,06]$ e maduro $[\mathrm{Z}(\mathrm{n}=$ 276) $=-0,1, \mathrm{p}=0,92]$.

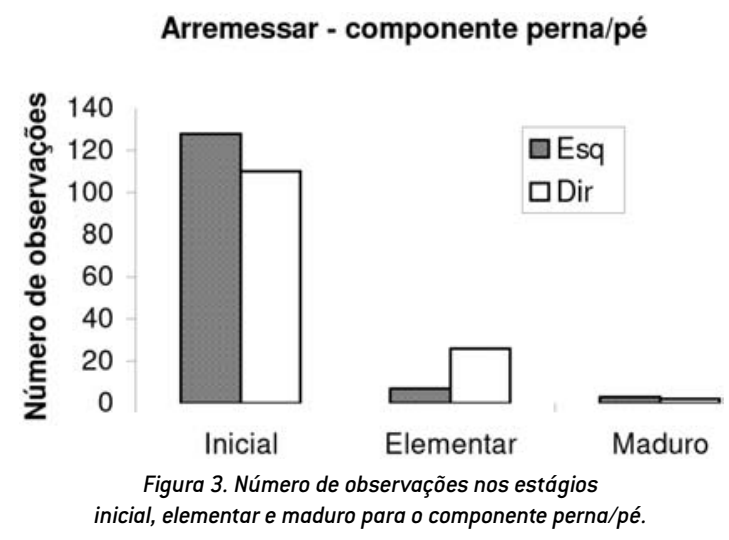

\section{Padrão fundamental chutar}

O número de observações encontradas no componente braço/tronco pode ser observado na Figura 4. A análise dos componentes mostrou diferença significativa entre as observações no estágio inicial [Z (n $=276)=-2,6, p<0,01]$, com um maior número de observações para o lado esquerdo. Não foi encontrada diferença significativa para as observações no estágio elementar $[Z(n=276)=0,73, p=0,47]$ e maduro $[Z(n=276)=1,66, p=0,09]$.

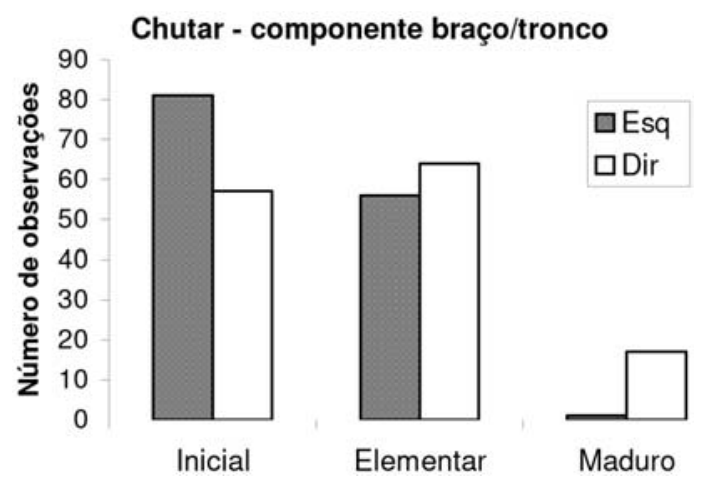

Figura 4. Número de observações nos estágios inicial, elementar e maduro para o componente braço/tronco.
O número de observações encontradas no componente perna pode ser observado na Figura 5. Na análise do padrão do componente perna observou-se uma diferença significativa entre as observações no estágio inicial $[\mathrm{Z}(\mathrm{n}=276)=-2,29, p<0,05] \mathrm{e}$ maduro $[Z(n=276)=2,7, p<0,01]$. Não houve diferença significativa para as observações no estágio elementar $[Z(n=276)=-0,62, p=0,53]$.

\section{Chutar - componente perna}

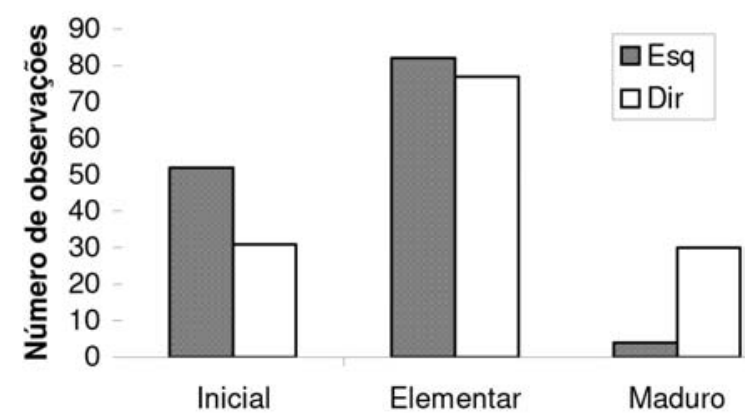

Figura 5. Número de observações nos estágios inicial, elementar e maduro para o componente perna.

\section{DISCUSSÃO E CONCLUSÃO}

O presente estudo teve como objetivo analisar os efeitos da assimetria lateral de desempenho nos padrões fundamentais arremessar e chutar em crianças. A análise geral dos resultados aponta para existência de assimetria de desempenho em todos componentes que compõem os padrões fundamentais chutar e arremessar. Esses resultados corroboram os achados de pesquisas anteriores realizadas com crianças $(9,10)$, evidenciando o efeito da assimetria de desempenho em infantis.

$\mathrm{Na}$ análise do componente braço da habilidade arremesso foi encontrado um maior número de observações no estágio elementar para o membro esquerdo, enquanto para o membro direito foi encontrado um maior número de observações no estágio maduro. Estes resultados indicam um maior nível de desenvolvimento no membro direito para o componente braço. Entendendo que o fenômeno da assimetria de desempenho tem um caráter dinâmico, é possível que a interação entre aspectos biológicos e ambientais seja o fator marcante para um padrão mais desenvolvido no membro direito. A predominância 
do hemisfério esquerdo no processamento dos aspectos seqüenciais e temporais da ação (2) favorece uma melhor execução do membro direito.

Somado a esse fator, a preferência manual das crianças que participaram desse experimento pela utilização do membro direito nas atividades recreativas e esportivas vivenciadas na escola apresenta um papel importante na emergência desses padrões assimétricos entre os membros contralaterais.

A assimetria encontrada nos componentes do padrão fundamental chutar indica que este efeito é também encontrado em movimentos manipulativos realizados com os membros inferiores em crianças. A análise de todos os componentes e ambas as habilidades mostra que, com exceção do componente braço do arremessar, o efeito de assimetria foi destacado no estágio inicial. Os membros esquerdo superior e inferior apresentaram um maior número de observações, quando comparados aos membros direitos. Esses resultados reforçam a existência de assimetrias entre os membros contralaterais inferiores e superiores nos padrões fundamentais e que os membros superior e inferior esquerdo apresentam um menor nível de proficiência nessa fase do desenvolvimento motor. Um outro ponto de análise é perceber em qual dos componentes das habilidades arremessar e chutar o efeito de assimetria foi melhor observado. Em ambos os padrões fundamentais os resultados mais claros foram aqueles relacionados ao membro diretamente ligado à execução, ou seja, o componente braço para o arremesso e o componente perna para o chute. No arremesso, o componente braço apresentou maior número de observações para o lado esquerdo no estágio inicial e para o lado direito no estágio maduro. Nos componentes que utilizam musculaturas sinergistas, componentes tronco e perna/pé, o efeito de assimetria lateral foi encontrado somente em um dos estágios. O mesmo efeito foi encontrado para o padrão fundamental chutar, tendo apenas o componente perna apresentado assimetria lateral de desempenho, tanto no estágio inicial quanto no maduro.

Nas habilidades arremessar e chutar estão envolvidos o controle de diferentes segmentos corporais que formam os componentes do movimento. Os resultados favoráveis aos membros direito encontrados no padrão de execução do componente braço, habilidade arremesso, e componente perna, habilidade chutar, podem estar relacionados aos resultados observados nos demais componentes. Como exemplo, se os componentes tronco e perna/pé se encontram em estágios iniciais de desenvolvimento, toda a estabilidade da habilidade arremessar pode ficar comprometida, gerando limitações na qualidade do movimento no braço esquerdo, estando este diretamente relacionado ao movimento. Os resultados do estudo de Teixeira e Gasparetto (10) não mostraram esse maior efeito da assimetria nos membros diretamente relacionados à manipulação. Novos estudos são necessários para investigar o efeito da assimetria nos componentes envolvidos no movimento.

Respeitando as limitações da análise quantitativa realizada nesse experimento, os resultados encontrados permitem inferir que o desenvolvimento dos padrões fundamentais das crianças não ocorre de uma forma global, ou seja, numa perspectiva bilateral. Sugerem-se algumas questões a serem levantadas para próximos estudos. Qual a relação entre a assimetria no padrão de movimento e a assimetria no desempenho do padrão? Em outras palavras, poderia um menor nível de força ou precisão de um determinado membro estar relacionado a um menor nível de desenvolvimento do padrão fundamental para aquele membro? Uma outra questão se refere à diminuição da assimetria entre membros nos padrões fundamentais devido à prática. Ou seja: é possível minimizar o déficit no desenvolvimento bilateral das crianças pela atuação do profissional da Educação Física? Entendendo as assimetrias como um fenômeno multifacetado que, entre outros, é influenciado pela prática, a manipulação da preferência lateral das crianças, recorrendo à adequação de regras e brincadeiras, poderia ser um fator de interferência nesse processo.

\section{CORRESPONDÊNCIA}

\section{Guilherme Lage}

Universidade FUMEC

FCS - Faculdade de Ciências da Saúde

Departamento de Educação Física

Rua da Paisagem, 240 - Vila da Serra

CEP 34000-000

Brasil

menezeslage@aol.com 


\section{REFERÊNCIAS}

1. Bryden PJ (2000). Lateral preference, performance, skilled behavior and task complexity. In Mandal M, BulmanFleming B, Tiwari G (eds.) Side Bias: a neuropsychological approach. Netherlands: Kluwer Academic Press.

2. Carson RG (1989). Manual asymmetries: feedback processing, output variability, and spatial complexity - resolving some inconsistencies. Journal of Motor Behavior 21: 38-47.

3. Corballis PM (2003). Visuospatial processing and the righthemisphere interpreter. Brain and Cognition 53: 171-176.

4. Coryell J (1985). Infant rightward asymmetries predict right-handedness in childhood. Neuropsychologia 23: 269-271.

5. Gallahue DL, Ozmun JC (2005). Compreendendo o desenvolvimento motor: Bebês, Crianças, Adolescentes e Adultos. São Paulo: Phorte.

6. Haywood KM (1993). Life span motor development. Champaign, Il.: Human Kinetics.

7. McClenaghan BA, Gallahue DL (1985). Movimientos fundamentales. Buenos Aires: Pan-americana.

8. Peters M (1981). Handedness: effect of prolonged practice on between hand performance differences. Neuropsychologia 19: 587-590.

9. Petrie BF, Peters M (1980). Handedness: Left/right differences in intensity of grasp response and duration of rattle holding in infants. Infants Behavior and Development 3: 215-221.

10. Teixeira LA, Gasparetto ER (2002). Lateral asymmetries in the development of the overarm throw. Journal of Motor Behavior 34: 151-160.

11. Teixeira LA, Chaves CEO, Silva MVM, Carvalho MA (1998). Assimetrias laterais no desempenho de habilidades motoras relacionadas ao futebol. Kinesis 20:77-92.

12. Vasconcelos O. (2004). Preferência lateral e assimetria motora funcional: uma perspectiva de desenvolvimento. In Barreiros J, Godinho M, Melo F, Neto C (eds.) Desenvolvimento e aprendizagem: perspectivas cruzadas. Lisboa: FMH. 